\begin{tabular}{|c|c|c|}
\hline $\mathrm{AO}$ & $\begin{array}{c}\text { European Association for the } \\
\text { Development of Renewable Energies, Environment } \\
\text { and Power Quality (EA4EPQ) }\end{array}$ & $\begin{array}{l}\text { International Conference on Renewable Energies and Power Quality } \\
\text { (ICREPQ'12) } \\
\text { Santiago de Compostela (Spain), 28th to 30th March, } 2012\end{array}$ \\
\hline
\end{tabular}

\title{
Medium-voltage distribution feeders in open-loop and closed-loop arrangement
}

\author{
G. Štumberger ${ }^{1}$, K. Deželak ${ }^{1}$, M. Rošer ${ }^{2}$, R. Škof ${ }^{2}$ and T. Kastelic ${ }^{3}$ \\ ${ }^{1}$ University of Maribor \\ Faculty of Electrical Engineering and Computer Science \\ Smetanova 17, 2000 Maribor (Slovenia) \\ Phone/Fax number:+386 2220 7075, e-mail: gorazd.stumberger@uni-mb.si, klemen.dezelak@uni-mb.si \\ ${ }^{2}$ Elektro Celje d.d. \\ Vrunčeva 2a, 3000 Celje (Slovenia) \\ Phone/Fax number:+386 3420 1000, e-mail: miran.roser@elektro-celje.si, robert.skof@elektro-celje.si \\ ${ }^{3}$ Elektro Primorska d.d. \\ Erjavčeva 22, 5000 Nova Gorica (Slovenia) \\ Phone/Fax number:+386 5339 6700, e-mail: tomaz.kastelic@elektro-primorska.si
}

\begin{abstract}
This paper deals with a medium-voltage distribution network. The feeders in the open-loop arrangement are changed to the closed-loop arrangement with the aim to improve reliability of the power supply. The paper focuses on the analysis of transient and steady-state operation in the closed-loop that is established by connecting feeders supplied from two different transformers located in the same transformer substation. The transformers are equipped with the tap changers that can change the amplitude of the $20 \mathrm{kV}$ voltages in steps whilst the phase cannot be changed. The possibilities to control the active and reactive power at both ends of the medium-voltage loop are discussed for the case when only the voltage amplitude can be changed and for the case when the voltage amplitude and phase angle can be changed. A simple algorithm for closed-loop active and reactive control is applied. The results of simulations and field testing performed in a $20 \mathrm{kV}$ network are presented.
\end{abstract}

\section{Key words}

Medium-voltage distribution network, operation in the loop, power flow control, field testing, simulation.

\section{Introduction}

In the medium-voltage networks, the feeder closed-loop arrangements are often used to improve reliability of power supply, to improve voltage profiles in the feeders with distributed generation, and to reduce losses caused by electric power transmission. According to [1], the closedloop arrangements of the feeders can be classified as the loops of the type I, type II and type III. In the type I loops, two feeders, supplied form the same transformer, are connected to form the loop. The two connected feeders, supplied from the two different transformers, located in the same substation, form the loop type II. When the feeders, supplied from the two different transformers located in the two different substations, are connected, the loop type III is established. The authors in [1] and [2] focus primarily on the loops of type I. They deal with the unbalance analysis and with the feasibility study for upgrading the feeders from radial open-loop arrangement to the closedloop arrangement type I. The focus of investigation in [3] and [4] is the control of loop distribution systems with distributed generation. The loop power flow control and voltage characteristics in a distribution system with distributed generation are presented in [5]. The authors in [6] investigate the possibility for reduction of line losses by upgrading the distribution system from normally radial to normally closed-loop arrangement of feeders.

The authors of this paper performed analysis, simulations and field testing for all three types of the closed-loop arrangements of feeders. However, the paper focuses only on the closed-loop type II. In the given case, the primary goal of the feeder rearrangement, from the openloop feeders to the closed-loop ones, is the improved reliability of power supply. It is substantial for supply of sensitive loads. The possibilities of improving voltage profiles, inclusion of distributed generation and power losses in the feeders are, in the given case, of secondary importance. Let us suppose that both feeders, that form the closed-loop, are sized in such a way that they can supply all loads on both feeders even when they are supplied only from the one side. Additionally, let us suppose that the feeders are equipped with the directional and delayed over-current and distance protection relays sensitive enough to properly open the closed-loop and eliminate only the line section with the fault. If all the aforementioned presumptions are fulfilled, the most important problem that remains and must be solved is the control of the active and reactive power at both ends of the closed-loop, which means at both feeders. This paper 
shows how the active and reactive power can be controlled in the closed-loop type II. A simple closed-loop active and reactive power control is presented for an ideal case where the voltage amplitude and phase can be changed continuously. Such a system, where the amplitude and phase of the transformer's output voltages could be changed independently and continuously, is not available. Therefore, only the results of simulation are given for this case. On the contrary, the results of simulations and field testing are given for the case of open-loop power control where the existing transformers equipped with tap changer units are applied. In this case the voltage amplitude can be changed only in the discrete voltage steps while the voltage angle cannot be influenced directly.

\section{Discussed system and control realization}

The discussed medium-voltage network is shown in Fig. 1. It consists of a substation Krško with two 40 MVA $110 \mathrm{kV} / 20 \mathrm{kV}$ Dyn transformers equipped with the tap changer units. They supply the busbars S1 and S2 whiles M3 and M4 are the measurement points. The two feeders connected to the busbars S1 and S2 are used to form the closed-loop type II. The network structure of the discussed feeders is schematically presented in Fig. 2, where the variable voltage sources are the transformers TR I and TR II shown in Fig. 1.

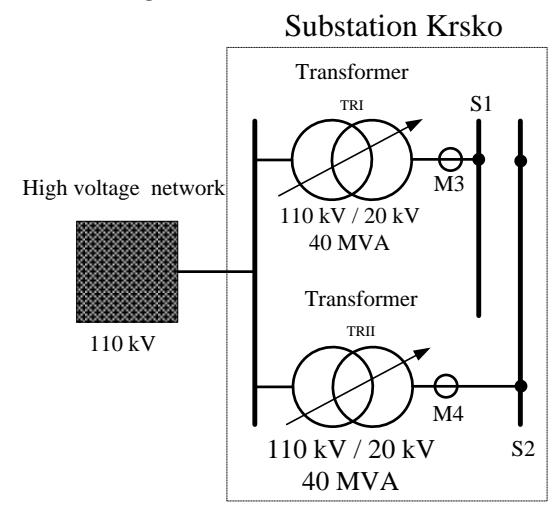

Fig. 1. Substation Krško

Let us first neglect all the loads connected to both feeders and all higher order harmonic components in the currents and voltages. In this case, the energy flow between both feeders in the closed-loop arrangement, given in the form of the active power $P$ and reactive power $Q$, is described by (1) and (2), respectively:

$$
\begin{aligned}
& P=\frac{X\left|U_{1}\right|\left|U_{2}\right|}{|Z|^{2}} \sin (\delta)+\frac{R\left|U_{1}\right|^{2}}{|Z|^{2}}-\frac{R\left|U_{1}\right|\left|U_{2}\right|}{|Z|^{2}} \cos (\delta) \\
& Q=\frac{X\left|U_{1}\right|^{2}}{|Z|^{2}}-\frac{X\left|U_{1}\right|\left|U_{2}\right|}{|Z|^{2}} \cos (\delta)-\frac{R\left|U_{1}\right|\left|U_{2}\right|}{|Z|^{2}} \sin (\delta)
\end{aligned}
$$

where $\left|U_{1}\right|$ and $\left|U_{2}\right|$ are the amplitudes of voltages at both end of the closed-loop, $\delta$ is the phase angle difference between both voltages, $Z$ is the impedance of the closedloop power line, whiles $R$ and $X$ are the resistance and reactance of the conductor. Neglecting the resistance $R$, equations (1) and (2) can be changed to (3) and (4).

$$
\begin{aligned}
& P=\frac{\left|U_{1}\right| \cdot\left|U_{2}\right|}{X} \cdot \sin (\delta) \\
& Q=\frac{\left|U_{1}\right|^{2}}{X}-\frac{\left|U_{1}\right|\left|U_{2}\right|}{X} \cos (\delta)
\end{aligned}
$$

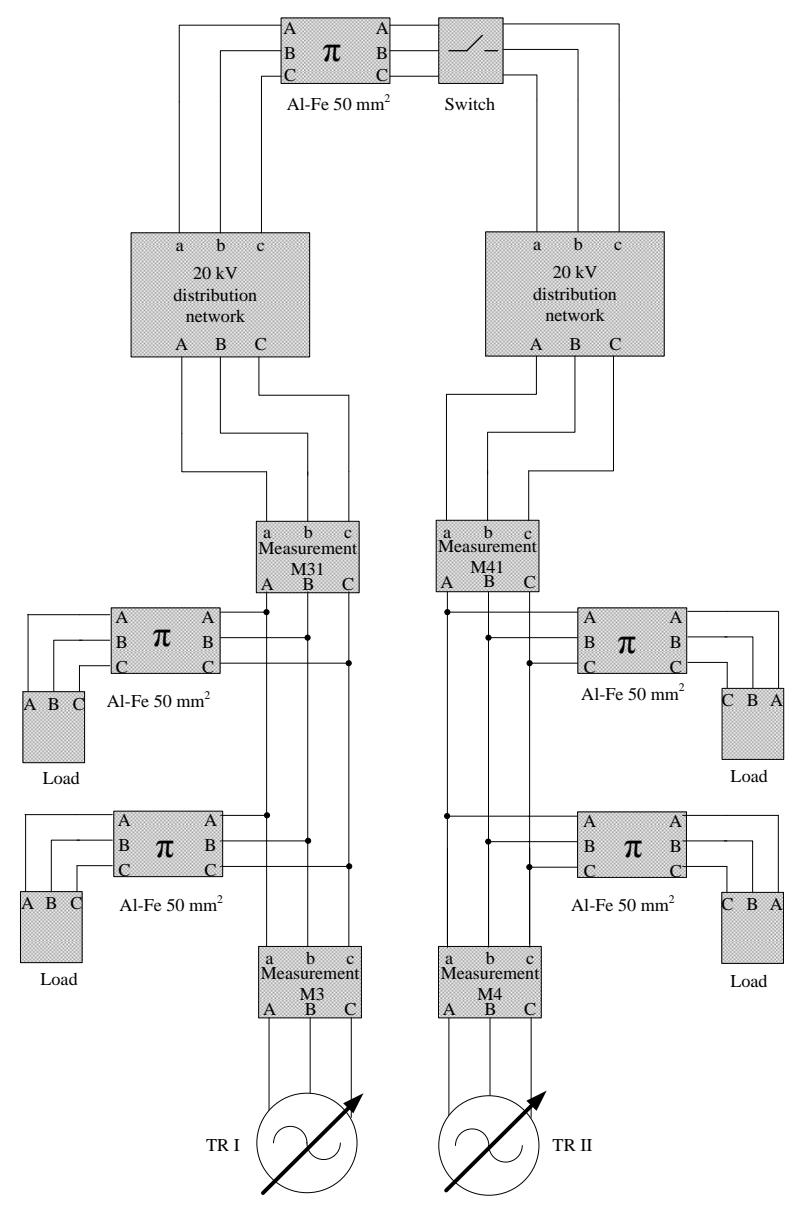

Fig. 2. Schematic presentation of discussed feeders

Both transformers shown in Fig. 1 are of the same size and type and both are supplied from the same $110 \mathrm{kV}$ busbar. Therefore, the difference $\delta$ in the phase angles of voltages on busbars S1 and S2, shown in Fig 1, can be considered as small, which leads to (5). Considering (5) and small changes around the given operating point, (3) and (4) change to (6) and (7):

$$
\begin{aligned}
& \delta \rightarrow 0 \Rightarrow \cos \delta \rightarrow 1 \Rightarrow \sin \delta \rightarrow \delta \\
& \Delta P=\frac{\left|U_{1}\right| \cdot\left|U_{2}\right|}{X} \cdot \delta \\
& \Delta Q=\frac{\left|U_{1}\right|^{2}}{X}-\frac{\left|U_{1}\right|\left|U_{2}\right|}{X}=\frac{\left|U_{1}\right|}{X}\left(\left|U_{1}\right|-\left|U_{2}\right|\right)
\end{aligned}
$$

where $\Delta P$ and $\Delta Q$ are the small changes in the active and reactive power around the operating point given by $P$ and $Q$. By introducing the difference between the amplitudes of both voltages $\Delta U(8),(6)$ and (7) change to (9) and (10).

$$
\begin{aligned}
& \left|U_{2}\right|=\left|U_{1}\right|+\Delta U \\
& \Delta P=\frac{\left|U_{1}\right| \cdot\left(\left|U_{1}\right|+\Delta U\right)}{X} \cdot \delta=\frac{\left|U_{1}\right|^{2}}{X} \cdot \delta+\frac{\left|U_{1}\right| \cdot \Delta U}{X} \cdot \delta
\end{aligned}
$$




$$
\Delta Q=\frac{\left|U_{1}\right|}{X}(-\Delta U)
$$

Equations (9) and (10) show, that the changes $\Delta P$ and $\Delta Q$ are not decupled. The reactive power can be changed by $\Delta U$ whiles the active power changes due to the $\Delta U$ and $\delta$. Based on these facts, a simple scheme for closed-loop control of the active and reactive power, shown in Fig. 3, is introduced. The realizations of the active and reactive power PI controllers are schematically shown in Figs. 4 and 5, where indices 'ref' and 'M" denote the reference and measured values, respectively. The initial conditions are marked with ZP, "lim" denotes the controller output limits whiles $K_{\mathrm{p}, \mathrm{P}}, K_{\mathrm{i}, \mathrm{P}}$ and $K_{\mathrm{p}, \mathrm{Q}}, K_{\mathrm{i}, \mathrm{Q}}$ are the parameters of the active and reactive power PI controllers.

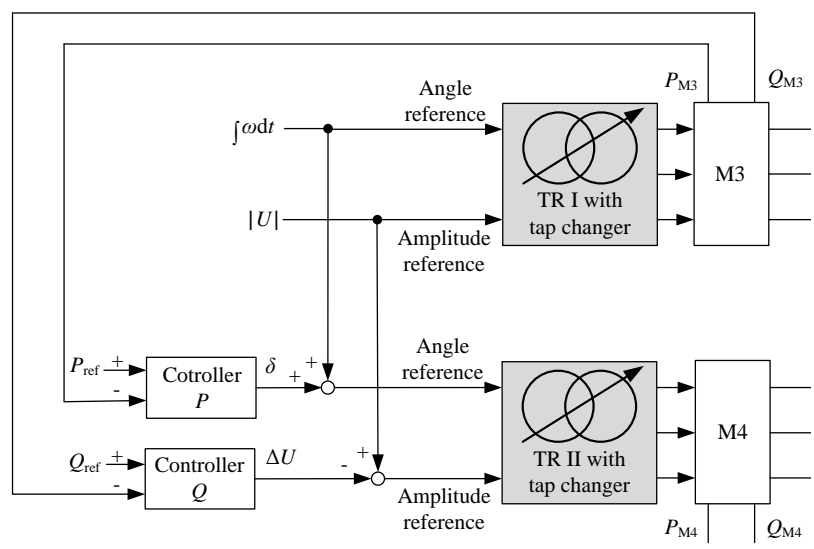

Fig. 3. Simple scheme for closed-loop control of the active power $P$ and reactive power $Q$

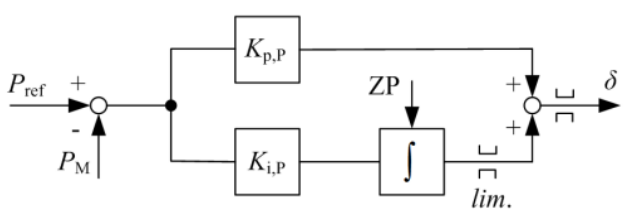

Fig. 4. Scheme presentation of the active power PI controller

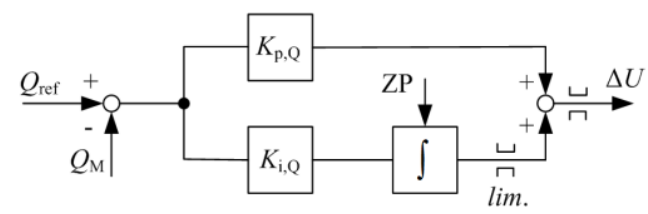

Fig. 5. Scheme presentation of the reactive power PI controller

The control structure for the closed-loop active and reactive power control was applied in simulations of the system presented in Figs. 1 and 2. The simulations were performed for the discussed system considering loads and without loads. The voltage sources with continuously changing voltage amplitude and phase angle were applied.

The field tests were performed on the system shown in Figs. 1 and 2 during normal operation. The open-loop feeders were rearranged to the closed-loop type II by switching-on the switch shown at the top of Fig. 2. Since only the discrete step changes in voltage amplitude can be achieved with the existing transformers with stepping tap changer units, only the open -loop power control was applied.

\section{Results}

Fig. 6 shows the simulation results for the closed-loop control of the active and reactive power. The loads shown in Fig. 2 are neglected. Presented are the reference active power $P_{\text {ref }}$ and reactive power $Q_{\text {ref }}$ as well as the active and reactive power in the measuring points M3 and M4, $P_{\mathrm{M} 3}, Q_{\mathrm{M} 3}$ and $P_{\mathrm{M} 4}, Q_{\mathrm{M} 4}$, respectively. They are shown in Figs. 1 and 3 . The amplitude and angle of the transformer's output voltages is changing continuously. The loads connected along the closed-loop are neglected. The active and reactive power $P_{\mathrm{M} 4}, Q_{\mathrm{M} 4}$, measured in the measurement point M4 and shown in Fig. 2, are equal to the sum of the active and reactive power $P_{\mathrm{M} 3}, Q_{\mathrm{M} 3}$, measured in the measurement point M4, shown in Fig. 2, and the power losses along the closed-loop between M3 and M4.
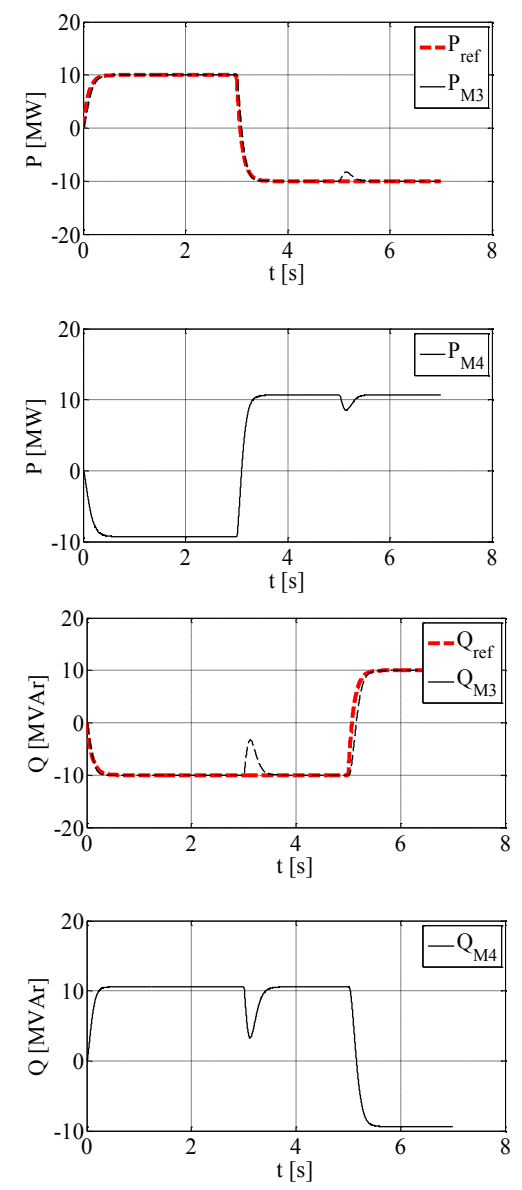

Fig. 6. Closed-loop control active and reactive powerneglected loads

Fig. 7 shows the same variables as Fig. 6. However, in the results shown in Fig. 7, the loads shown in Fig. 2 are considered, which leads to the increased differences between the active and reactive powers in the measurement points M4 and M3.

The results presented in Figs. 6 and 7 clearly show that the control scheme described in this paper enables closed-loop control of the active and reactive power. It is shown that $P_{\mathrm{M} 3}$ and $Q_{\mathrm{M} 3}$ follow their references in the cases with and without load along the closed-loop between the measurement points M3 and M4. 
Unfortunately, the available equipment is not able to change the amplitude and angle of the transformer's output voltage. The existing transformers with tap changers can change only the voltage amplitude in discrete voltage steps which is achieved by changing the tap changer setting point.

Figs. 8 and 9 show the results of field testing and simulations in the case of step change in the tap changer setting for one step. Presented are the time behaviors of the line voltage $u_{4}$, line current $i_{4}$, active power $P_{4}$ and reactive power $Q_{4}$ measured in M4 shown in Fig. 2.
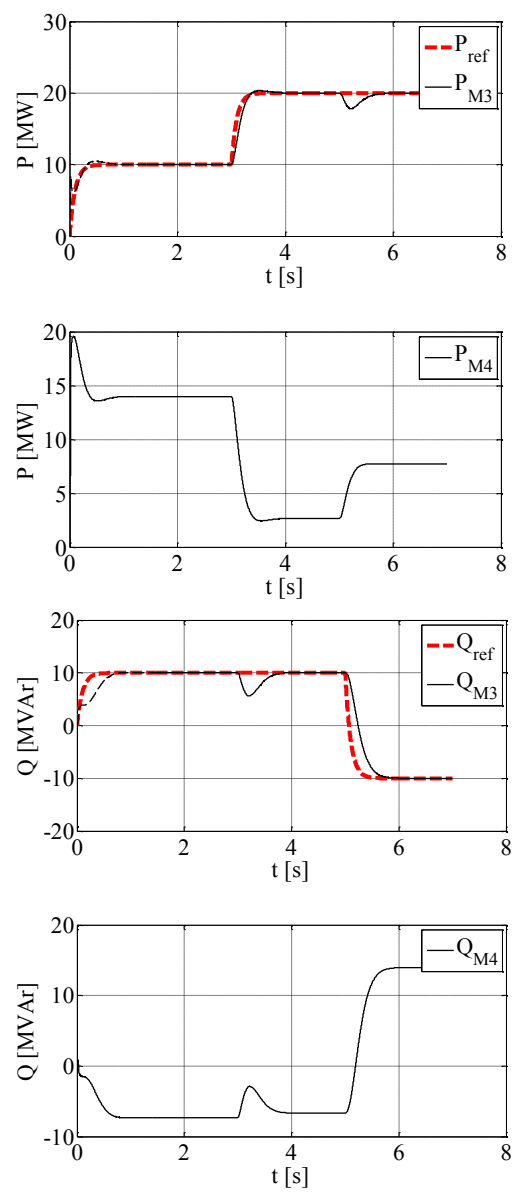

Fig. 7. Closed-loop control active and reactive powerconsidered loads
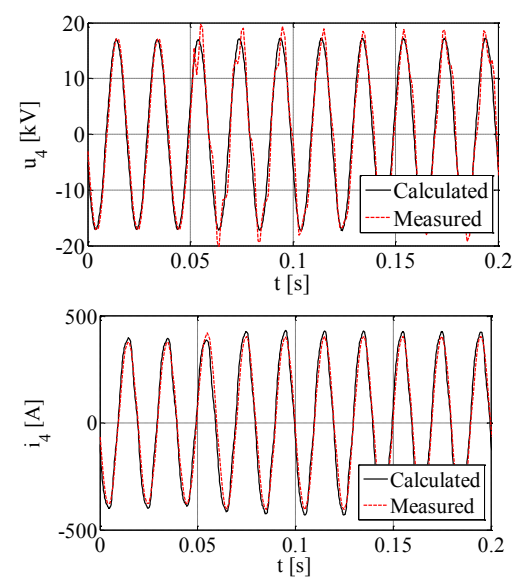

Fig. 8. Results of field testing and simulations: the time behaviors of the line voltage $u_{1}$ and current $i_{1}$ for voltage increase due to the change in tap changer setting for one step.
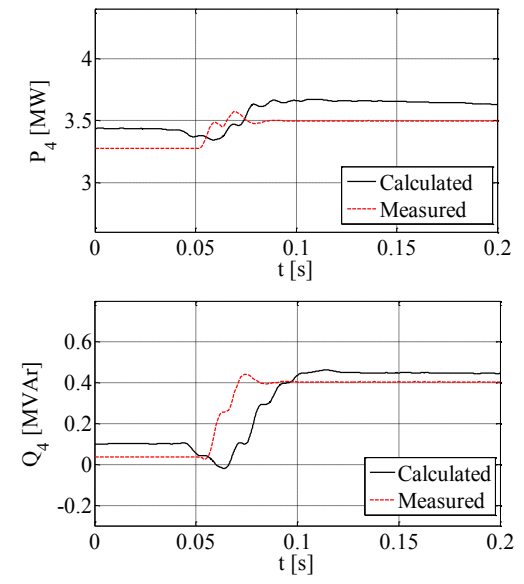

Fig. 9. Results of field testing and simulations: the time behaviors of the active power $P_{1}$ and reactive power $Q_{1}$ for voltage increase due to the change in tap changer setting for one step

\section{Conclusion}

The paper deals with the closed-loop arrangement of feeders in the medium-voltage distribution network. The closed-loop arrangement is established to improve reliability of power supply. It is shown that the control scheme described in this paper makes possible closedloop control of the active and reactive power when feeders are in the closed-loop arrangement. The existing transformers with tap changers enable only discrete step changes in the voltage amplitude which is insufficient for realisation of the proposed control.

\section{Acknowledgement}

This work was partially supported by the ARRS project no. P2-0115 and V2-1023.

\section{References}

[1] T. H. Chen, W. T. Huang, J. C. Gu, G. C. Pu, Y. H. Hsu, T. Y. Guo, Feasibility Study of Upgrading Primary Feeders from Radial and Open-Loop to Narmally Closed-Loop Arrangement, IEEE Transactions on Power Systems, vol. 19, no. 3, pp. 1308 1316, 2004.

[2] N. Okada, Verification of Control Method for a Loop Distribution System using Loop Power Flow Controller, in Proc. Power Systems Conference and Exposition (PSCE'06), pp. 2116 - 2123, 2006, Atlanta.

[3] W. C. Yang, W. T. Huang, J. Z. Chen, Unbalanced Analysis of Changing Medium-Voltage Distribution Fedders from Open-Loop to Closed-Loop Arrangement, in Proc. Third International Conference on Innovative Computing Information and Control, 2008, Dalian, China.

[4] Y. Hanai, Y. Hayashi, J. Matsuki, Voltage Control for a Loop Distribution System with Renewable Energy Sources, in Proc. International Conference on Renewable Energies and Power Quality (ICREPQ), 2010, Spain.

[5] N. Okada, H. Kobayashi, K. Takigawa, M. Ichikawa, K. Kurokawa, Loop power flow control and voltage characteristics of distribution system for distributed generation including pv system, 3rd World Conference on Photovoltoic Energy Conversion, May 11-18, Osaka, Japan, pp. 2284-2287, 2003.

[6] W.-T. Huang and S.-T. Chen, Line loss reduction by distribution system upgrading from radial to normally closedloop arrangement, Ninth International Conference on Hybrid Intelligent Systems, pp. 334-338, 2009. 